\title{
Metabolic Syndrome Biomarkers in Type II Diabetic Ethiopian Patients
}

Fitsum Girma Tadesse ${ }^{1^{*}}$, Yesehak Worku ${ }^{2}$, Yeweyenhareg Feleke ${ }^{3}$ and Tarek H. El-Metwally ${ }^{4}$

${ }^{1}$ Department of Medical Microbiology, Radboud Institute for Molecular Life Sciences, Route 268, Geert Grooteplein 26-28, Nijmegen 6525 GA, Netherlands

${ }^{2}$ Department of Biochemistry, College of Medicine, Addis Ababa University, Addis Ababa, Ethiopia

${ }^{3}$ Department of Internal Medicine, Faculty of Medicine, Addis Ababa University, Addis Ababa, Ethiopia

${ }^{4}$ Faculty of Medicine, Assiut University, Assiut, POB: 71526, Egypt

"Corresponding author: Fitsum Girma Tadesse, Department of Medical Microbiology, Radboud Institute for Molecular Life Sciences, Route 268, Geert Grooteplein 26-28, Nijmegen 6525 GA, Netherlands, Tel: 251-912-627-540; Fax: 251-111-552-350; E-mail: fitsezemichael@gmail.com, fitsum.girma@aau.edu.et

Received date: March 31, 2014, Accepted date: April 23, 2014, Published date: May 02, 2014

Copyright: (c) 2014 Tadesse FG, et al. This is an open-access article distributed under the terms of the Creative Commons Attribution License, which permits unrestricted use, distribution, and reproduction in any medium, provided the original author and source are credited.

\section{Abstract}

Background: Insulin resistance, which precedes by many years the onset of and accompanies type II diabetes (T2D), is strongly associated with a clustering of cardiovascular risk factors, termed metabolic syndrome (MetS).

Objectives: The aim of this study was to investigate the extent of MetS risk in T2D Ethiopian patients.

Methods: A total of 72 T2D patients and 20 normal healthy controls without MetS were studied. Based on the world health organization criteria, $59.72 \%(43 / 72)$ of the patients had MetS.

Results: Type 2 diabetic patients with MetS $(0.953 \pm 0.007)$ were significantly $(P<0.01)$ obese than those without $(0.913 \pm 0.012)$. Plasma total triglyceride (TG) $(206.9 \pm 16.91)$ and blood pressure $(138.8 \pm 3.247)$ were strongly significantly high $(\mathrm{P}<0.001)$ in patients with MetS as compared to those without MetS $(104.4 \pm 6.766$ and $122.8 \pm$ 2.725, respectively). On the other hand, plasma high density lipoprotein-cholesterol (40.91 \pm 3.070$)$ and total peroxide $(0.055 \pm 0.001)$ were found at a significantly $(P<0.05)$ lower amounts in patients with MetS as compared to those without $(50.90 \pm 2.601$ and $0.052 \pm 0.001$, respectively). In patients with MetS, the IR index showed a significant association $(P<0.05)$ with the dyslipidemia markers: TG $(r=0.254)$, low density lipoprotein-cholesterol $(r=0.262)$, and total cholesterol $(r=0.320)$. Waist-hip circumference ratio showed a positive significant correlation with blood pressure $(r=0.330, P<0.05)$ and $C$-reactive protein was strongly associated with serum insulin concentration $(r=0.382, P<0.01)$.

Conclusion: Ethiopian T2D patients with MetS are at a greater risk of hypertensive, dyslipidemia and oxidative stress states, and developing cardiovascular disorders. Interventions should be planned to help those patients avoid/delay onset of cardiovascular complications anticipated upon the accumulation of predisposing factors that are components of MetS.

Keywords: Metabolic syndrome; Type II diabetes; C-reactive protein; Total peroxides; Dyslipidemia; Insulin resistance; Central obesity

\section{Introduction}

Type II diabetes (T2D) is a complex and progressive disease that is strongly associated with obesity and Insulin Resistance (IR) as its main characteristic features[1]. Insulin resistance is an early event and precedes the onset of T2D by many years [2]. However, during its early stage IR is compensated by hyperinsulinemia, thus preserving normal glucose tolerance. Deterioration into impaired glucose tolerance occurs when either IR increases or the insulin secretory response of the $\beta$-cells decreases, or both [3]. This is partly induced by the apparent hyperglycemia that translates into progressive deterioration and degranulation of $\beta$-cells, often accompanied by a decreased $\beta$-cell mass [1].

Insulin resistance coexists not only with $\mathrm{T} 2 \mathrm{D}$ but also with a constellation of cardiovascular risk factors that world health organization (WHO) designated as metabolic syndrome (MetS) [4].
Insulin resistance is thought to be the mechanism driving the other components of MetS [5]. Furthermore, MetS is accompanied by a proinflammatory and prothrombotic state [6] that enhances the potential for acute thrombosis through hypercoagulability and impaired fibrinolysis [7] to finally precipitate cardiovascular disease (CVD). An emerging body of evidence documents associations of elevated low-grade inflammation markers such as $\mathrm{C}$-reactive protein (CRP) concentrations in individuals with IR [8].

On the other hand, IR can be induced and aggravated by increased oxidative stress (OxS), which is common in T2D [9]. Association of IR with OxS resembles a vicious circle. Oxidative stress increases IR through interference with intracellular insulin signaling [10]. Chronic hyperinsulinemia secondary to IR can, in turn, be responsible for the generation of free radicals and decrease in antioxidant enzyme activity $[9,11]$. Given the risk of CVD to diabetic patients, there is a need to investigate the underlying alteration in the constellation of components of MetS in T2D patients in order to improve recognition of individuals at risk of future CVD and alleviate the complications patients could face. Studies have evaluated this association among 
other ethnic groups. However, to our knowledge, there was no report on the Ethiopian T2D patients. We, therefore, sought to evaluate the underlying alterations in Ethiopian T2D patients with MetS.

\section{Materials and Methods}

The study proposal was reviewed and approved by the Ethical Review Committee of the Faculty of Medicine of Addis Ababa University. Permission to conduct the study was also obtained from the department of Internal Medicine. Before starting the study, all voluntary participants were given an explanation on the purposes and methods of the study. Informed written consent was obtained from each study participant prior to his/her enrolment in the study.

This project applied a cross-sectional study design and conducted at Tikur Anbessa Specialized Hospital, Faculty of Medicine, Addis Ababa University, Addis Ababa, Ethiopia. A total of 72 T2D patients (36 men and 36 women) regularly attending the diabetic clinic participated in the study. Socio-economically matching 20 (12 males and 8 females) normal healthy subjects were included in the study. They were staff members and post-graduate students of the faculty with normal BMI and without MetS (Table 1).

\begin{tabular}{|c|c|c|c|}
\hline Parameter & Control $(\mathbf{n}=\mathbf{2 0})$ & Patient $(\mathbf{n}=\mathbf{7 2})$ & $\begin{array}{l}\text { P values < } \\
\text { (Unpaired t-test })\end{array}$ \\
\hline BMI (Kg/m2) & $22.55 \pm 0.698$ & $25.56 \pm 0.470$ & 0.01 \\
\hline WC $(\mathrm{cm})$ & $83.05 \pm 2.660$ & $96.05 \pm 1.214$ & 0.001 \\
\hline HC $(\mathrm{cm})$ & $94.65 \pm 1.864$ & $102.7 \pm 1.197$ & 0.01 \\
\hline WHR & $0.87 \pm 0.014$ & $0.937 \pm 0.007$ & 0.001 \\
\hline SBP $(\mathrm{mmHg})$ & $118.50 \pm 1.957$ & $132.4 \pm 2.404$ & 0.01 \\
\hline DBP $(\mathrm{mmHg})$ & $75.00 \pm 1.539$ & $81.21 \pm 1.138$ & 0.01 \\
\hline FBG $(\mathrm{mg} / \mathrm{dL})$ & $76.45 \pm 2.775$ & $180.8 \pm 7.562$ & 0.001 \\
\hline CRP $(\mathrm{mg} / \mathrm{dL})$ & $1.48 \pm 0.208$ & $21.7 \pm 4.605$ & 0.05 \\
\hline HDL-C $(\mathrm{mg} / \mathrm{dL})$ & $61.35 \pm 3.721$ & $44.95 \pm 2.176$ & 0.01 \\
\hline LDL-C $(\mathrm{mg} / \mathrm{dL})$ & $50.83 \pm 6.599$ & $89.65 \pm 5.753$ & 0.05 \\
\hline TG $(\mathrm{mg} / \mathrm{dL})$ & $147.40 \pm 11.410$ & $161 \pm 10.190$ & NS \\
\hline Insulin $(\mathrm{mU} / \mathrm{L})$ & $4.75 \pm 0.288$ & $5.205 \pm 0.212$ & NS \\
\hline $\begin{array}{c}\text { TP }(\mu M) \\
\text { HOMA-IR }(\mathrm{mU} / \\
\text { L.mM })\end{array}$ & $0.051 \pm 0.001$ & $0.054 \pm 0.001$ & NS \\
\hline
\end{tabular}

Table 1: Anthropometric, clinical and biochemical characteristics assessment of the study Ethiopian Type II diabetic patients and healthy controls. Abbreviations: $\mathrm{BMI}=$ Body Mass Index; DBP = Diastolic Blood Pressure; CRP $=$ C-Reactive Protein; FBG $=$ Fasting Blood Glucose; HC = Hip Circumference; HDL-C = High Density Lipoprotein-Cholesterol; HOMA-IR $=$ Homeostasis Model Assessment-Insulin Resistance; LDL-C = Low Density LipoproteinCholesterol; SBP $=$ Systolic Blood Pressure; TG $=$ Triglycerides; $\mathrm{TP}=$ Total Peroxide; $\mathrm{WC}=$ Waist Circumference; WHR $=$ Waist-Hip Circumference Ratio; NS = Non-Significant Difference. Data presented are means \pm SEM.
After an overnight fasting $5 \mathrm{~mL}$ blood was drawn from antecubital vein of the arm of each study participant into anti-coagulant free blood collection vacutainer tubes. The whole blood was left to stand vertically for 30 minutes on ice and the serum from the sample was processed and collected by centrifugation at $3000 \mathrm{rpm}$ for 10 minutes at $4^{\circ} \mathrm{C}$. The separated serum was aliquot stored at $-70^{\circ} \mathrm{C}$ until further analysis.

Medical history (age, sex, drugs, smoking, alcohol consumption, and duration of diabetes) and anthropometric measurements (height, weight, waist, and hip circumference) were taken. Waist circumference (WC) was measured with a soft measuring tape on standing subjects midway between the lowest rib and the iliac crest to the nearest $0.1 \mathrm{~cm}$ at minimal respiration. Hip circumference $(\mathrm{HC})$ was taken at the greatest gluteal protuberance to the nearest $0.1 \mathrm{~cm}$. The $\mathrm{WC}-\mathrm{HC}$ ratio was calculated to get the WHR. Weight was measured with a lever balance, to the nearest $100 \mathrm{~g}$, while subjects wore light clothing without shoes. Height was measured, to the nearest $0.5 \mathrm{~cm}$, without shoes, with a measuring tape, with eyes looking straight ahead, with a right-angle triangle resting of the scalp against the wall. Body mass index (BMI) was calculated as weight in kilogram divided by height in meter square expressed in $\mathrm{kg} / \mathrm{m}^{2}$.

Blood Pressure measurements were taken on the right arm twice after the subject had been seated in supine position for at least 5 minutes, with a standard sphygmomanometer, and the mean blood pressure value in $\mathrm{mmHg}$ was used. A questionnaire was administered for each participant, both patients and normal controls, to collect other demographic details.

Fasting blood glucose (FBG), high density lipoprotein-cholesterol (HDL-C), total triglyceride (TG) were assayed by enzymatic methods (Biocon" Diagnostik, Vöhl-Marienhagen, Germany). C-reactive protein was assayed with a quantitative turbidimetric immunoassay (Linear Chemicals, S.L., Barcelona, Spain). Insulin was assayed with a solid phase two-site immunoassay (Mercodia AB, Uppsala, Sweden). Total peroxide (TP) concentration was determined using the ferricxylenol orange "FOX" method [12]. Low density lipoproteincholesterol (LDL-C) was determined using Friedewald's equation [13].

The present study used the WHO criteria to identify patients with MetS [5]. Homeostasis model assessment of IR (HOMA-IR) was used for measurement of insulin sensitivity [14].

To avoid confounding by exogenously taken insulin, patients under insulin therapy were excluded. Further, patients with liver disease, thyroid disease, Cushing's syndrome, alcoholics, smokers, those taking lipid lowering drugs, patients suffering from arthritis, and patients with HIV on anti-retroviral drug were excluded.

Information obtained from questionnaire and laboratory analyses was analyzed using Prism 3.0 package (GraphPad Software, Inc, San Diego, CA, USA) and Microsoft Excel 2003. The minimum level of statistical significance was set at $\mathrm{p}<0.05$. The data was expressed as Mean \pm SEM. Results were analyzed statistically using column statistics and t-test for comparison of unpaired two-tailed variables. Group differences were determined by analysis of variance (ANOVA) with post hoc testing using the Newman-Keuls method. Correlation among the investigated parameters in each group was tested by the non-parametric Spearman's analysis. 


\section{Results}

Table 1 shows that means of anthropometric and clinical parameters were significantly different between healthy controls and T2D patients in general. Biochemical investigation of serum FBG, CRP, HDL-C and LDL-C revealed significant mean differences between control groups and T2D patients. Differences in mean serum TG, TP, and insulin concentration remained insignificantly high in T2D patients, however. The mean age of the controls and patients was $35.45 \pm 1.019$ and $54.85 \pm 1.242$, respectively.

Table 2 presents ANOVA results on the differences in the mean values of investigated parameters in healthy control subjects as compared to T2D patients sub grouped on the basis of MetS, with and without.

\begin{tabular}{|c|c|c|c|}
\hline Parameter & Control vs. Without & Control vs. With & $\begin{array}{l}\text { Without vs. } \\
\text { With }\end{array}$ \\
\hline WHR & 0.05 & 0.001 & 0.01 \\
\hline HDL-C & 0.05 & 0.001 & 0.05 \\
\hline SBP/DBP & NS & 0.001 & 0.001 \\
\hline TG & NS & 0.01 & 0.001 \\
\hline TP & NS & 0.05 & 0.05 \\
\hline CRP & 0.05 & 0.01 & NS \\
\hline LDL-C & 0.01 & 0.05 & NS \\
\hline HOMA-IR & 0.001 & 0.001 & NS \\
\hline
\end{tabular}

Table 2: Anthropometric, clinical and biochemical characteristics assessment of the study Ethiopian Type II diabetic patients stratified by metabolic syndrome as compared to healthy control groups. Abbreviations: DBP $=$ Diastolic Blood Pressure; CRP $=$ C-Reactive Protein; HDL-C = High Density Liporotein-Cholesterol; HOMA-IR = homeostasis model assessment-Insulin Resistance; LDL-C = Low Density Liporpotein-Cholesterol; SBP = Systolic Blood Pressure; TG = Triglycerides; $\mathrm{TP}=$ Total Peroxide; $\mathrm{WHR}=$ Waist-Hip Circumference Ratio; NS $=$ non-significant difference; Values are means \pm SEM.

Comparing between all the three groups (Healthy controls, patients without MetS and patients with MetS), waist-hip circumference ratio (WHR) $(0.876 \pm 0.014)$ was significantly lower in controls than that of patients without MetS $(0.913 \pm 0.012 ; \mathrm{P}<0.05)$ and with MetS $(0.953 \pm$ 0.007; $\mathrm{P}<0.001$ ). Comparing the two patients' subgroups (with vs. without MetS) revealed a significant difference $(\mathrm{P}<0.01)$ too. High density lipoprotein-cholesterol control level $(61.35 \pm 3.740 \mathrm{mg} / \mathrm{dL})$ was significantly higher than patients without MetS $(50.90 \pm 2.601$ $\mathrm{mg} / \mathrm{dL}, \mathrm{P}<0.05)$ and patients with MetS $(40.91 \pm 3.070 \mathrm{mg} / \mathrm{dL}$; $\mathrm{P}<0.001)$. Comparing patients' subgroups revealed a significant difference $(\mathrm{P}<0.05)$.

Systolic blood pressure (SBP), TG and TP resulted in insignificant differences when comparing healthy controls with T2D patients without MetS. However, the controls' level of SBP (118.5 \pm 1.957 $\mathrm{mmHg}$ ) was significantly lower than that of patients with MetS (138.8 $\pm 3.247 \mathrm{mmHg}, \mathrm{P}<0.001)$, with very strong significant difference $(\mathrm{P}<0.001)$ between patient subgroups. Total TG in healthy control subjects $(147.4 \pm 11.41)$ was significantly lower than patients with MetS $(206.9 \pm 16.91 \mathrm{mg} / \mathrm{dL} ; \mathrm{P}<0.01)$. Comparison of patients' subgroups revealed a highly significant difference $(\mathrm{P}<0.001)$. Serum
TP in healthy control volunteer subjects $(0.051 \pm 0.001)$ as compared to that of patients with MetS were significantly lower $(0.056 \pm 0.001$; $\mathrm{P}<0.05)$.

On the other hand, HOMA-IR, CRP and LDL-C revealed insignificant differences between patients' subgroups. Healthy controls' IR index $(0.913 \pm 0.079)$ was strongly significantly lower $(\mathrm{P}<0.001)$ as compared to that of patients without $(2.148 \pm 0.259)$ and with MetS $(2.434 \pm 0.169)$. Serum CRP in healthy volunteer subjects $(1.480 \pm 0.208 \mathrm{mg} / \mathrm{dL})$ was significantly lower than patients without MetS $(21.31 \pm 7.926 \mathrm{mg} / \mathrm{dL})$ and patients with MetS $(21.880 \pm 5.651$ $\mathrm{mg} / \mathrm{dL}), \mathrm{P}<0.05$ and $\mathrm{P}<0.01$ respectively. The values of $\mathrm{LDL}-\mathrm{C}$ in healthy subjects $(50.83 \pm 6.599 \mathrm{mg} / \mathrm{dL})$ was significantly lower than each of patients without MetS $(98.52 \pm 9.744 \mathrm{mg} / \mathrm{dL})$ and with MetS $(81.53 \pm 6.733 \mathrm{mg} / \mathrm{dL}), \mathrm{P}<0.01$ and $\mathrm{P}<0.05$ respectively.

In a correlation analyses in T2D patients with MetS, IR state significantly positively $(\mathrm{P}<0.05)$ associated with each of the dyslipidemia markers; TG ( $\mathrm{r}=0.254)$, LDL-C $(\mathrm{r}=0.262)$ and TC $(\mathrm{r}=0.320)$. Waist-hip circumference ratio significantly positively $(\mathrm{P}<0.05)$ correlated with each of the SBP $(\mathrm{r}=0.259)$ and DBP $(\mathrm{r}=0.330)$. C-reactive protein revealed a strongly significant positive $(\mathrm{P}<0.01)$ correlation with serum insulin concentration $(r=0.382)$.

Among parameters investigated in $\mathrm{T} 2 \mathrm{D}$ patients population as a whole serum insulin concentration revealed a significant negative $(\mathrm{r}=-0.243, \mathrm{P}<0.05)$ association with HDL-C and a positive significant association with WC $(\mathrm{r}=0.409, \mathrm{P}<0.001)$ and DBP $(\mathrm{r}=0.238, \mathrm{P}<0.05)$. WHR showed a significant positive correlation with SBP $(r=0.288$, $\mathrm{P}<0.01)$ and DBP $(\mathrm{r}=0.329, \mathrm{P}<0.01)$. Total triglyceride showed a significant negative correlation with $\mathrm{SBP}(\mathrm{r}=0.273, \mathrm{P}<0.05), \mathrm{DBP}$ $(\mathrm{r}=0.341, \mathrm{P}<0.01)$, and $\mathrm{TP}(\mathrm{r}=0.271, \mathrm{P}<0.05)$. Insulin resistance revealed a significant positive correlation with DBP $(r=0.239, \mathrm{P}<0.05)$, CRP $(r=0.221, \mathrm{P}<0.05)$, and TG $(\mathrm{r}=0.260, \mathrm{P}<0.05)$. C-reactive protein showed a significant negative association with TAC $(r=-0.243$, $\mathrm{P}<0.05)$.

\section{Discussion}

Type II diabetes patients with MetS in the present study were significantly obese as compared to those without MetS. In addition to that, dyslipidemia, an increase in circulating TG and a low level in HDL-C, characterized them. In the association study, dyslipidemia was highly correlated with IR index of patients with MetS. The current dominant paradigm is that IR leads to dyslipidemia although it is still possible that dyslipidemia may also cause IR [15]. The vicious "dyslipidemia-IR-hyperinsulinemia" cycle has been forwarded to explain this phenomenon [16].

It is not all the possible list of dyslipidemia, however, that could possibly be causal factors or results of IR. Instead, dyslipidemia consisting of high TGs and low HDL-C is a widely recognized lipid pattern that is frequently associated with IR and subsequent development of chronic heart disease (CHD). Furthermore, in Framingham Heart Study incident CHD risk that is associated with low plasma HDL-C or hypertriglyceridemia was significantly increased only in the presence of IR [17]. Taken together, these results implicate that the study subjects with MetS in this study are at increased risk of developing CVD, as they had hypertriglyceridemia and low HDL-C.

Although there are several causes of CVD, atherosclerosis (leading to CHD) and/or hypertension are the most common ones. The dyslipidemic and obesity state in this study is accompanied by a 
Page 4 of 5

pronounced increase in blood pressure in patients with MetS. Excess weight gain contributes to increased blood pressure, partly due to activation of the sympathetic nervous system in the kidney that results in increased renal sodium reabsorption and impaired pressure natriuresis, which appears to be mediated in part by increased levels of the adipocyte-derived hormone leptin [18].

There are emerging biochemical explanations for the association of dyslipidemia with CHD, in the presence of IR. In a hyperlipidemic animal model an increased ROS generation and an over expression of the NADPH oxidase gp91phox subunit have been demonstrated [19]. This indicates that the patients in the present study were at increased risk of developing CHD as they had elevated TP in their sera. Currently, OxS is considered as a novel component of the MetS. Assessment of plasma OxS may contribute to identify a subset of MetS patients at increased cardiovascular risk, candidates to more intensive therapies [20]. Previous study reported higher OxS level in subjects with more MetS components [21].

The OxS in diabetics is possibly mediated to a significant extent via increased production of ROS from the high glucose, possibly in concert with fatty acids. Oxidative stress activates a number of cellular stress response pathways [10]. These pathways lead to both IR and impaired insulin secretion. Reports highlighted that central obesity, IR and dyslipidemia significantly correlate with antioxidant enzymes activity [21]. Although all body cells are bathed in the hyperglycemic state in T2D, endothelial cells are extremely vulnerable. Exposure of endothelial cells to high glucose leads to augmented production of superoxide anion, which may quench nitric oxide, a potent endothelium-derived vasodilator that participates in the general homeostasis of the vasculature [22]. With all these effects increases in OxS may contribute to impaired vascular function, inflammation, thrombosis, and atherosclerosis and ultimately give rise to vascular disease [23].

In addition to OxS, subjects with MetS have a higher inflammation status [24]. In clinical studies it is confirmed that inflammation contributes towards the early stages of CHD [25].Correlation analysis, in the present study, revealed that serum insulin concentration is strongly correlating with CRP which is in line with previous reports. In Peruvian adults elevated CRP was significantly associated with increased mean fasting insulin [8]. In addition, the level of CRP was found higher in U.S. youth who had the MetS[26].One of the mechanisms that could explain the inflammation that was observed in the present study could be obesity state of the patients. A study in Portuguese implicated an increase in inflammation (level of CRP) with severity of obesity and high blood pressure [27].The Nod Like Receptor (NLR) family of innate immune cell sensors like the Nlrp3 inflammasome senses obesity-associated 'danger-signals', such as ceramide. Nlrp3 contributes to obesity-induced inflammation and IR [28]. This is worsened by the accumulation of macrophages that secrete proinflammatory mediators [29]. All culminate into a combination of altered functions of insulin target cells; such as in adipose tissue, liver, and skeletal muscle [30].

Above all the OxS and inflammation states are cross-talking with one another and could ultimately exert devastating effects. Inflammation increases the production of ROS resulting in an increased OxS with over-activation of NADPH oxidase [31]. This process reduces the bioavailability of NO. On the other hand, OxS alters the nature, pattern, and magnitude of cytokines produced. With all these effects OxS could play the central role in the alterations observed in patients with MetS increased. This is in agreement with a
Japanese report which indicated that $\mathrm{OxS}$ in accumulated fat is an important pathogenic mechanism of obesity-associated MetS [32].

Taken together, in our Ethiopian T2D patients, central obesity, followed by dyslipidemia and low-grade inflammation and $\mathrm{OxS}$, are the main pathogenic mechanism underlying the metabolic changes observed in the study subjects with MetS that predisposes T2D patients to hypertension and risk of CVD. In a proof, patients without MetS had comparable state of blood pressure, lipid profile, and OxS as compared to controls. Since patients with the MetS have greater risk of developing the CVD, this study can help make proper guidelines for prevention and screening, at early time, in T2D Ethiopian patients.

\section{Acknowledgement}

The study was financially supported by Addis Ababa University

\section{References}

1. Kahn SE, Hull RL, Utzschneider KM (2006) Mechanisms linking obesity to insulin resistance and type 2 diabetes. Nature 444: 840-846.

2. Evans JL, Goldfine ID, Maddux BA, Grodsky GM (2002) Oxidative stress and stress-activated signaling pathways: a unifying hypothesis of type 2 diabetes. Endocr Rev 23: 599-622.

3. Poitout V, Robertson RP (2008) Glucolipotoxicity: fuel excess and betacell dysfunction. Endocr Rev 29: 351-366.

4. Metascreen Writing Committee, Bonadonna R, Cucinotta D, Fedele D, Riccardi G, et al. (2006) The metabolic syndrome is a risk indicator of microvascular and macrovascular complications in diabetes: results from Metascreen, a multicenter diabetes clinic-based survey. Diabetes Care 29: 2701-2707.

5. Stolar M (2007) Metabolic syndrome: controversial but useful. Cleve Clin J Med 74: 199-202, 205-8.

6. Deen D (2004) Metabolic syndrome: time for action. Am Fam Physician 69: 2875-2882.

7. Wannamethee SG, Lowe GD, Shaper AG, Rumley A, Lennon L, et al. (2005) The metabolic syndrome and insulin resistance: relationship to haemostatic and inflammatory markers in older non-diabetic men. Atherosclerosis 181: 101-108.

8. Gelaye B, Revilla L, Lopez T, Suarez L, Sanchez SE, et al. (2010) Association between insulin resistance and c-reactive protein among Peruvian adults. DiabetolMetabSyndr 2: 30.

9. Sarafidis PA, Grekas DM (2007) Insulin resistance and oxidant stress: an interrelation with deleterious renal consequences? J CardiometabSyndr 2: 139-142.

10. Evans JL, Goldfine ID, Maddux BA, Grodsky GM (2003) Are oxidative stress-activated signaling pathways mediators of insulin resistance and beta-cell dysfunction? Diabetes 52: 1-8.

11. Bloch-Damti A, Bashan N (2005) Proposed mechanisms for the induction of insulin resistance by oxidative stress. Antioxid Redox Signal 7: 1553-1567.

12. Harma M, Harma M, Erel O (2005) Measurement of the total antioxidant response in preeclampsia with a novel automated method. Eur J ObstetGynecolReprodBiol 118: 47-51.

13. Friedewald WT, Levy RI, Fredrickson DS (1972) Estimation of the concentration of low-density lipoprotein cholesterol in plasma, without use of the preparative ultracentrifuge. ClinChem 18: 499-502.

14. Matthews DR, Hosker JP, Rudenski AS, Naylor BA, Treacher DF, et al. (1985) Homeostasis model assessment: insulin resistance and beta-cell function from fasting plasma glucose and insulin concentrations in man. Diabetologia 28: 412-419.

15. Reaven G (2012) Insulin resistance and coronary heart disease in nondiabetic individuals. ArteriosclerThrombVascBiol 32: 1754-1759. 
Citation: Tadesse FG, Worku Y, Feleke Y, El-Metwally TH (2014) Metabolic Syndrome Biomarkers in Type II Diabetic Ethiopian Patients. J Metabolic Synd 3: 1000139. doi:10.4172/2167-0943.1000139

Page 5 of 5

16. Li N, Fu J, Koonen DP3, Kuivenhoven JA3, Snieder H4, et al. (2014) Are hypertriglyceridemia and low HDL causal factors in the development of insulin resistance? Atherosclerosis 233: 130-138.

17. Robins SJ, Lyass A, Zachariah JP, Massaro JM, Vasan RS (2011) Insulin resistance and the relationship of a dyslipidemia to coronary heart disease: the Framingham Heart Study. ArteriosclerThrombVascBiol 31: 1208-1214.

18. Hall JE, da Silva AA, do Carmo JM, Dubinion J, Hamza S, et al. (2010) Obesity-induced hypertension: role of sympathetic nervous system, leptin, and melanocortins. J BiolChem 285: 17271-17276.

19. Maeda K, Yasunari K, Sato EF, Inoue M (2005) Enhanced oxidative stress in neutrophils from hyperlipidemic guinea pig. Atherosclerosis 181: 87-92.

20. Hopps E, Noto D, Caimi G, Averna MR (2010) A novel component of the metabolic syndrome: the oxidative stress. NutrMetabCardiovasc Dis 20: 72-77.

21. Yubero-Serrano EM, Delgado-Lista J, Peña-Orihuela P, Perez-Martinez P, Fuentes F, et al. (2013) Oxidative stress is associated with the number of components of metabolic syndrome: LIPGENE study. Exp Mol Med 45: e28.

22. Giugliano D, Ceriello A, Paolisso G (1996) Oxidative stress and diabetic vascular complications. Diabetes Care 19: 257-267.

23. Berliner JA, Navab M, Fogelman AM, Frank JS, Demer LL, et al. (1995) Atherosclerosis: basic mechanisms. Oxidation, inflammation, and genetics. Circulation 91: 2488-2496.

24. Chen SJ, Yen CH, Huang YC, Lee BJ, Hsia S, et al. (2012) Relationships between inflammation, adiponectin, and oxidative stress in metabolic syndrome. PLoS One 7: e45693.
25. Luc G, Bard JM, Juhan-Vague I, Ferrieres J, Evans A, et al. (2003) Creactive protein, interleukin-6, and fibrinogen as predictors of coronary heart disease: the PRIME Study. ArteriosclerThrombVascBiol 23: 1255-1261.

26. Ford ES, Ajani UA, Mokdad AH; National Health and Nutrition Examination (2005) The metabolic syndrome and concentrations of Creactive protein among U.S. youth. Diabetes Care 28: 878-881.

27. Santos AC, Lopes C, Guimarães JT, Barros H (2005) Central obesity as a major determinant of increased high-sensitivity C-reactive protein in metabolic syndrome. Int J Obes (Lond) 29: 1452-1456.

28. Vandanmagsar B, Youm YH, Ravussin A, Galgani JE, Stadler K, et al. (2011) The NLRP3 inflammasome instigates obesity-induced inflammation and insulin resistance. Nat Med 17: 179-188.

29. Olefsky JM, Glass CK (2010) Macrophages, inflammation, and insulin resistance. Annu Rev Physiol 72: 219-246.

30. Schenk S, Saberi M, Olefsky JM (2008) Insulin sensitivity: modulation by nutrients and inflammation. J Clin Invest 118: 2992-3002.

31. Rivera J, Sobey CG, Walduck AK, Drummond GR (2010) Nox isoforms in vascular pathophysiology: insights from transgenic and knockout mouse models. Redox Rep 15: 50-63.

32. Furukawa S, Fujita T, Shimabukuro M, Iwaki M, Yamada Y, et al. (2004) Increased oxidative stress in obesity and its impact on metabolic syndrome. J Clin Invest 114: 1752-1761. 